Mateja Cerovšek

Doctorante

Faculté des Lettres, Université de Ljubljana

Slovénie

vodomka@gmail.com
UDK [811.133.1'42:796]:070

DOI: 10.4312/vestnik.13.109-126

\title{
L'IMITATION DE LA CONVERSATION EN TANT QUE STRATEGIE DE CONNIVENCE DANS LE DISCOURS JOURNALISTIQUE SPORTIF
}

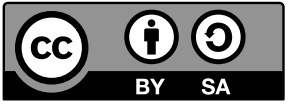

\section{INTRODUCTION}

Tout discours journalistique répond à un contrat communicationnel. Celui-ci s'inscrit dans le cadre de la communication médiatique qui est, en principe, monologale. Il existe, en effet, un certain décalage spatio-temporel entre la communication sur un événement et sa réception, et où, faute de pouvoir répondre, le destinataire n'est pas un vrai interlocuteur (Boyer : 31). Par manque de contact réel, « les moyens de communication de masse [ont] très vite ressenti le besoin de créer un semblant d'échange avec leur public » (Boyer : 31). La presse, surtout, met en place des approches dans son discours afin d'atténuer cet " écart discursif» (Chovanec 2008 : 220) et ne pas perdre son lectorat. Il lui importe de créer l'impression d'une communauté imaginaire en s'appuyant, pour l'assurer, sur la connivence, qui correspond dans le domaine journalistique à un rapport imaginé, « de réduction de la distance constitutive entre le journal et son lecteur » (Herman 2008 : 184).

Dans le discours journalistique sportif(DJS), la place de la connivence s'explique tout autant par le type de discours, soit le discours journalistique, que par sa thématique telle qu'elle est perçue dans la société. La sociologie fonctionnaliste a défini le sport au-delà d'un phénomène de divertissement. Dans son rôle d'institution sociale, le sport assure, entre autres, une fonction socio-émotive et une fonction socio-intégrative (Stevenson et Nixon 1972 ; Delaney 2015), provoquant chez ses spectateurs des émotions plus ou moins fortes et alimentant des sentiments de camaraderie et d'appartenance à une communauté qui se forment autour d'une même passion partagée. Derèze (2009 : 92) remarque d'ailleurs : « Pratiquants, spectateurs, supporteurs, amateurs, ils ont, pour la plupart, une histoire commune avec le sport. » Il s'ensuit un certain attachement au sport de tous les sujets concernés au sein du discours journalistique sportif, créant une communauté sportive imaginaire.

Cet attachement au sujet sportif complémenté par le sentiment de camaraderie se traduit dans le DJS sous forme de connivence, et ceci par le biais des approches tant interdiscursives que linguistiques. Dans l'analyse du DJS britannique et français, Vanoudheusden 
(2010) évoque l'emploi de la terminologie et du jargon sportifs, des acronymes et des surnoms, ce qui couvre le « sociolecte sportif». Indispensable pour parler du sport, il démarque ainsi un même terrain de jeu pour ses adeptes qui s'attendent à « une discussion entre les spécialistes » (Vanoudheusden 2010 : 161). La connivence opère de même à un niveau interdiscursif, par le biais de références et d'allusions historico-culturelles du monde sportif que partagent le journaliste et le lecteur (Vanoudheusden 2010). Ce dernier se sentira davantage sollicité s'il est engagé dans une réception active. Chovanec (2008b) montre comment des procédés insistant sur la forme, tels que certains tropes, des jeux de mots et différentes allusions culturelles, mobilisent le destinataire, attirant son attention sur la forme du texte. Ils sont interactifs dans le sens où « ils demandent de la participation des lecteurs pour dévoiler le sens caché et l'apprécier » (Chovanec 2008b : 222). Ces moyens qui mettent en avant la forme et le plaisir de lire contribuent à atténuer l'écart discursif et le caractère impersonnel de la communication médiatique.

À des fins de connivence, on constate encore dans le DJS l'insertion de stratégies conversationnelles. Selon Fowler (1991), le discours de la presse use des démarches qui le rapprochent d'un discours oral afin de masquer qu'il relève aussi bien du divertissement que du discours institutionnel dont il est une voix. Lewandowski (2012) observe pourtant le phénomène dans le commentaire sportif direct en ligne. À l'instar du commentaire télévisé, ce genre naissant recourt à un vocabulaire informel et à l'insertion d'interrogation dans le discours afin « d'établir une relation plus personnelle avec ses lecteurs » (Lewandowski 2012 : 74). Dans le même genre hybride, Chovanec (2009) constate que l'introduction d'éléments oraux contribue à y créer l'illusion d'un pseudo-dialogue, renforçant en même temps le lien social entre les participants, soit le journaliste et les lecteurs-commentateurs.

L'objectif du présent article sera d'étudier davantage la notion de connivence journaliste-lecteur telle qu'elle est déployée dans le DJS de la presse française. Une analyse qualitative vise à montrer que la connivence en tant que stratégie discursive du DJS découle effectivement de l'impression voulue d'une communauté sportive. Ainsi nous concentrerons-nous sur l'identification des stratégies linguistiques de conversation qui, par l'imitation d'un échange souvent informel, soutiennent la dimension cohésive du sujet sportif. Menée à des niveaux lexical, syntaxique et discursif, l'analyse évoquera deux axes des stratégies conversationnelles, à savoir l'interactionnel et l'informel. Cela nous permettra d'estimer lequel de ces aspects de la conversationnalisation a plus de poids dans le DJS français contemporain, en proposant ainsi un nouvel angle pour les étudier simultanément.

\section{2} STRATEGIES LINGUISTIQUES DE CONVERSATION

L'objet de recherche en analyse conversationnelle française peut « en principe s' appliquer à tous les textes produits naturellement dans les situations réelles d'interaction sociale » 
(Bange 1983 : 3). Selon une convention générale, cependant, la conversation correspond plus précisément à un échange spontané. Dans le but d' « échanger les paroles », elle rassemble deux locuteurs ou plus qui se trouvent, du moins dans la situation donnée, sur un pied d'égalité, alternant les rôles de locuteur et d'allocutaire. Alternent aussi les tours de paroles de même que peuvent varier les sujets abordés dont l'enchaînement est immédiat et non prédéterminé (Traverso 2003).

Il existe une autre tradition de compréhension de la notion, au sein de laquelle « [1]e terme de 'conversation' s'emploie d'ordinaire seulement pour désigner un échange de propos sur un ton familier » (Bange 1983 : 3). C'est-à-dire que la caractéristique de la conversation rime avec la spontanéité, l'oralité, l'informalité du discours. Suivant cette conception, Fairclough (1992) a introduit dans son analyse critique du discours la notion de conversationnalisation qui se résume, globalement, à une tendance dans le discours public vers un style oralisé et informel, contrairement à la formalité traditionnellement prescrite à la communication publique. D'un côté, cette tendance sert à masquer le fondement institutionnel des discours publics : alors que l'écrit insinue « autorité, institution », l'oral dit « intime, familier, confidentiel » (Fowler 1991). De l'autre côté, en adoptant un style oralisé et informel les discours tels que celui de la presse se rapprochent de leurs lecteurs, se positionnant par le choix du registre sur un pied d'égalité.

La conversationnalisation se manifeste à tous les niveaux d'analyse : graphologique, lexical, morphosyntaxique et discursif. Dans le DJS, des études récentes l'ont tracée surtout dans le lexique du commentaire numérique en direct qui, oscillant entre les caractéristiques d'un commentaire oral en direct et des narrations sportives écrites, active un point d'interaction avec les lecteurs. Chovanec (2009), par exemple, analyse des éléments oralisés dans ce genre de DJS qu'il trouve présents à tous les niveaux, et notamment sous forme de lexique informel. Dans le même genre, l'emploi de tours de parole est constaté, révélant des marques conversationnelles qui relèvent d'une logique de la conversation strictement parlée (Lewandowski 2012). Par ailleurs, Lewandowski (2012 : 74) observe que : «Ce que le registre du commentaire en ligne a en commun avec le commentaire télévisé, ce sont quelques conventions de genre dont l'objectif général est d'établir une relation plus personnelle avec ses lecteurs. Ceci est accompli par les moyens de salutations et de clôture [de l'énoncé] de même que par l'emploi d'un vocabulaire informel et de questions occasionnelles. \ Ce lien personnalisé s'apparente à la connivence journalistique, et à la fois sportive. Elle se manifeste par une même volonté d'informaliser le discours de la presse sportive où : " L'écrit oralisé [y] est d'autant plus accepté que les lecteurs et les journalistes partagent 'le même bain sportif', celui du stade » (Vanoudheusden $2010: 195$ ).

Étudier davantage les stratégies conversationnelles dans le DJS de la presse française permettra de les contextualiser au-delà de la tendance d'informalisation des discours, soit dans le cadre de la connivence journaliste-lecteur. L'aspect informel étant souvent le seul à être mis en avant, nous inclurons dans notre étude tout autant le côté interactionnel 
des approches conversationnelles dans le DJS français, afin d'estimer lequel y est plus productif dans l'imitation d'une communauté sportive. Comme l'interactionnel se manifeste aussi au-delà du lexique, nous analyserons également le DJS français sous la loupe syntaxique et discursive.

3 METHODOLOGIE

Dans le but de relever des stratégies d'imitation de la conversation pour établir un lien de connivence entre le journaliste et ses lecteurs, nous avons mené une analyse qualitative d'un corpus de textes du DJS français. Nous avons puisé dans le corpus de textes français que nous avions créé entre novembre 2018 et octobre 2019. Le corpus est constitué d'articles sportifs qui sont parus sur les sites Internet des quotidiens généraux Le Figaro et Le Monde et du quotidien sportif L'Équipe.

Une première analyse qualitative avait été effectuée au cours de la sélection des articles. Cela nous a permis d'identifier des stratégies conversationnelles et, par la suite, d'extraire du corpus les textes pertinents pour la présente analyse. 25 articles ont été ensuite soumis à une analyse qualitative approfondie. Pour mieux comprendre l'enjeu interactionnel dans le DJS, nous y avons étudié le rôle des phrases interrogatives au niveau syntaxique. Au niveau discursif, nous avons observé deux caractéristiques constitutives de la conversation : les marqueurs discursifs qui la structurent et l'orientent, et les pronoms personnels avec leur fonction conative.

\section{$4 \quad$ ANALYSE}

\subsection{Niveau lexical}

Nous avons d'abord étudié la fonction d'insérer dans le DJS des formes appartenant au discours oral et des lexèmes d'un registre familier. On les apparente à un style oralisé du DJS de la presse qui se veut proche du commentaire sportif transmis en direct (Vanoudheusden 2010 : 194). Chovanec (2009) y reconnait un trait dont sont habituellement dotés les tabloïdes, et qui contribue à ce que s'estompent les barrières entre la communication publique et privée. Il remarque en même temps que : «L'effet général de ces expressions demeure dans la création d'une atmosphère où les lecteurs puissent se sentir à l'aise » (Chovanec 2009 : 199). Ces formes sont donc d'usage dans le DJS afin de s'approcher du lecteur. Tel est le cas dans (1) où l'adjectif hallucinant imite un commentaire qu'on produirait à l'oral, d'autant plus précédé de l'adverbe absolument. L'enthousiasme du journaliste transcrit dans ses propos, apparemment sans se soucier du langage, l'approche des lecteurs avec qui il estime partager le même sentiment. 
(1) Dee Bost (16 pts, 8 rbds, 4 p.d.) et ses coéquipiers ont signé un premier quart-temps absolument hallucinant, dans lequel les Grecs n'ont pas inscrit le moindre panier, se contentant de 6 points sur la ligne des lancers ! (EQ 14.11.19 Basketball)

Malgré le caractère formel du DJS, des formes telles que ça (2), truc (3), ou bien boulette (4) pour erreur, témoignent davantage d'une tendance à y insérer du lexique informel. Dans la presse sportive, on recourt effectivement à une variation de registres pour réduire l'écart discursif journaliste-lecteur qui reflète, d'après Fowler (1991), la difficile conciliation entre l'institutionnel et le personnel. Ce mélange particulier d'une voix institutionnelle et d'une autre plus personnalisée prend la forme d'un « idiome public » (Stuart Hall dans Fowler 1991 : 48), langue propre à chaque média qui la crée tout en prenant en compte le public auquel il s'adresse.

(2) Chaban est en transe. Ça tombe bien, ses favoris aussi. (EQ 31.12.18 Rugby)

(3) D'accord, les Bordelais avaient déjà fait le coup du succès bonifié dans les dernières secondes contre Lyon ( $8^{\mathrm{e}}$ journée, 35-13). Mais il s'est passé quelques trucs entre-temps, à commencer par le limogeage de Rory Teague. (F 4.1.19 Handball)

(4) Car la défense de l'OL est retombée dans ses travers, entre un Jason Denayer, capitaine d'un soir, apathique sur les deux buts toulousains, et un Anthony Lopes auteur d'un but contre son camp après avoir été déjà coupable d'une boulette fatale sur le terrain de Benfica en Ligue des champions (défaite 2-1). (M 4.11.19 Football)

Certains passages (5) multiplient des mots et des locutions familiers : l'emploi métaphorique de crâne pour dire tête ou pensées; la tronche et non pas le visage, la locution en carton au lieu de inintéressant. Tout se trouve dans la même phrase qu'une incidente (semble-t-il) et une forme verbale au passé simple. Que les différents registres s'entremêlent, incluant des formes typiques de l'oral et de l'écrit respectivement, est une caractéristique du DJS. Son mélange de registres attesté (Vanoudheusden 2010), le DJS combine les registres soutenu et familier, bien souvent dans une même phrase. Dans (6), le participe passé du verbe larguer se trouve ainsi à deux mots seulement d'une forme au passé simple, celui-ci étant la marque d'un style soutenu, voire littéraire.

(5) Ah, certains devaient les apercevoir, le hamac de la journée de repos, la sieste baveuse, le vidage de crâne devant une série Netflix en carton, mais c'était compter sans les experts en climatisation, qui avaient semble-t-il un chantier à 
finir et décidèrent de ventiler la tronche de tout le monde à trente-quatre bornes de l'arrivée. (EQ 16.7.19 Cyclisme)

(6) Il y a un mois, au Mugello, Rossi, largué aux essais $\left(18^{\mathrm{e}}\right)$ tomba rapidement en course. (EQ 28.6.19 MotoGP)

Au niveau lexical, on aperçoit encore dans le DJS français des procédés qui agissent sur la forme des mots. L'apocope, coupure d'une ou de plusieurs syllabes à la fin d'un mot, est le procédé de troncation le plus commun en français (Antoine 2000 : xxxv). Les motivations d'utiliser des troncats vont d'une simple économie de la langue et d'une accélération de la communication (au sein d'une profession, par exemple) jusqu'à freiner délibérément la compréhension en dehors d'un groupe.

(7) Un Monfils, sans jus et sans idée, plus tragique que magique dans la Caja Magica face à Yoshihito Nishioka, $73^{\mathrm{e}}$ mondial, beaucoup plus impliqué et auteur d'une belle perf' (7-5, 6-2). À bout de souffle à Bercy, la Monf' n'a visiblement pas profité de sa semaine londonienne en tant que remplaçant au Masters pour retrouver sa forme du début de saison. (F 20.11.19 Tennis)

Les troncations observées dans (7) ont une double intention : une volonté de banalisation mêlée à un fonctionnement identitaire. Montrer qu'une expression n'a plus de secret est l'une des raisons pour voir les troncats se multiplier dans la langue familière (souvent liée au langage d'un métier ou à un autre sociolecte). ${ }^{1}$ Pouvoir employer perf' au lieu de performance confirme la familiarité et l'aisance ressenties des deux participants discursifs au sein de la sphère sportive. Le second cas, la Monf, est le résultat d'avoir tronqué le nom propre du joueur de tennis français Gaël Monfils. Il part d'une motivation en partie identitaire (en reconnaissant dans l'apocope le mot initial, on démontre ses connaissances sportives) et, d'autre part, ludique et hypocoristique. La reprise de l'apocope qui circule parmi les suiveurs du tennis permet d'instaurer le sentiment de partager le même code.

\subsection{Niveau syntaxique}

Sur le plan syntaxique, nous observons comment le choix du type de phrase contribue à l'activation de la connivence dans le DJS. La phrase injonctive, par exemple, est par

1 «L'adoption de troncats, voire même leur création en langue familière, signale la banalisation du concept qu'ils désignent, l'apprivoisement du mot par le locuteur, une sorte de démystification du concept par la simplification du mot. » (Antoine 2000 : $\underline{\text { Liii) }}$ 
définition orientée vers l'allocutaire. ${ }^{2}$ Comme l'injonction ne peut pas fonctionner sans interlocuteur, son insertion dans le DJS (8) est une stratégie pour s'orienter vers le lecteur.

(8) Mais ne dites pas à Valérie Garnier que tous les feux sont au vert pour que la France empoche cette première place du groupe B, qui l'enverrait directement à Belgrade et en quarts de finale jeudi prochain. (EQ 30.6.19 Basketball)

Il en va de même pour les phrases interrogatives dont l'insertion mène elle aussi à amoindrir l'écart discursif dans le DJS (Chovanec 2009). En plus d'une valeur informative, on reconnaît aux phrases interrogatives une valeur interactionnelle : « L'interrogation, souvent appréhendée comme une tournure phrastique, a une force illocutoire très proche de l'exhortation de la communication simple. Elle permet d'attirer l'attention et de faire réagir l'interlocuteur. » (Bentounsi 2017 : 91)

Les questions rhétoriques représentent une des approches possibles pour imiter l'interaction dans un discours de communication à sens unique. Quoique sans réaliser l'acte d'interrogation « classique », c'est-à-dire émettre une question dans un but informatif, la question rhétorique connaît des usages au-delà d'un emploi stylistique : on lui admet de posséder une valeur argumentative, orientant le discours vers une conclusion voulue. Tel est le cas des interrogations fictives qui apparaissent à la clôture des commentaires sportifs écrits (Preoteasa 2017). Or, la question rhétorique sert de plus à interpeller le lecteur car « il est question d'une fonction émotive, celle-ci portant sur le message adressé aux allocutaires dans un dialogue fictif. Le discours devient ainsi orienté, l'assemblage du dispositif énonciatif trahissant des positions personnelles » (Preoteasa 2017 : 236). De nombreux exemples indiquent que le journaliste oriente sa question vers son lecteur, son allocutaire non présent, et ce n'est pas toujours d'une volonté argumentative qu'il part. Positionnées à la fin de l'article, les interrogatives (9) partent d'une vraie lacune d'informations. Il semble que, dans le cadre d' « un dialogue fictif » effectivement, ces formes d'interrogation partielle incitent le lecteur, sinon à prendre la parole réellement, du moins à réagir par la pensée.

(9) Mais est-ce raisonnable d'y croire ? Ce XV de France qui vient d'aligner quatre victoires pour la première fois depuis la Coupe du monde 2015 semble encore trop fragile, trop incertain, trop inconstant, pour ambitionner de terrasser un XV de la Rose sûr de ses forces, pragmatique et impitoyable. (F 8.10.19 Rugby)

Ces questions sont rhétoriques dans le sens où elles apparaissent dans un type de communication à sens unique. Elles ne sont pas pour autant simplement fictives ni

2 D'une fonction conative et dotée d'une force illocutoire, elle influence et agit sur l'allocutaire sous forme d' « ordre strict, conseil, souhait, prière, demande polie... » (Riegel, Pellat et Rioul 2001 : 407). 
oratoires $^{3}$; l'interrogative dans (9) étant la plus proche d'une question oratoire. Dans le cas ci-dessus, le journaliste se tourne vers son lecteur. Par l'action même de poser la question, il admet la présence de son interlocuteur fictif tout en se dévoilant lui-même. On parvient à « créer un rapport interactionnel et affectif entre le journaliste et le lecteur. Ainsi, [la question rhétorique] affiche la présence, énonce les positions et les jugements cachés de l'énonciateur derrière ses propos, comme elle trace la présence de l'énonciataire à qui on fait appel » (Bentounsi $2017: 100)$.

Nous avons constaté dans notre corpus un type de phrase interrogative qui semble à cheval entre une question rhétorique et une intervention fictive. Dans l'exemple (10), deux questions totales se succèdent. Leur objectif n'est pas de se renseigner, elles sont sans «force illocutoire d'une demande d'informations » (Jacques 1981 : 78). Elles cherchent plutôt à exprimer de l'incrédulité ; comme si elles reprenaient les propos de l'interlocuteur pour les contester.

(10) Les Lions de la Teranga n'ont pas de mental, et manquent de réalisme ? Ils ne savent plus gagner un match à élimination directe ? (F 6.7.19 Football)

Jacques (1981) appelle ce type d'interrogative une « question-écho ». Répéter la question précédemment émise, en totalité ou en partie, « traduit, sinon la non-compréhension, du moins une modalité de doute sur tel ou tel terme utilisé par le locuteur » (Jacques 1981 : 77). Par une « reprise» fictive du propos, on a l'impression de se trouver, soudainement, au milieu d'une conversation déjà en cours. La forme des deux questions renforce l'impression d'une interaction : relevant d'un registre familier, la phrase interrogative à l'intonation correspond plus à un discours oral qu'écrit. Ceci rapproche le propos d'une interaction orale où l'un des locuteurs vient de réagir à l'énoncé précédent.

Il arrive que le journaliste sportif pose une question et y réponde immédiatement, ôtant ainsi au lecteur la possibilité de réagir. Pour Bentounsi (2017 : 95), lorsque cette démarche est utilisée dans le discours journalistique, elle ne sert pas à solliciter mais à s'assurer l'attention de l'interlocuteur. Mais, comme dans (11), l'unité entière rappelle l'écho d'un propos suivi par la réaction du locuteur qui a maintenant (re)pris le tour, ce qui apporte une dynamique conversationnelle. L'exemple (11) donne en effet l'impression d'une interaction où le journaliste vient de reprendre le propos du lecteur-interlocuteur pour y répondre lui-même.

(11) Rageant? Sans doute. (EQ 20.10.19 Rugby)

Les phrases interrogatives jouent donc leur propre rôle dans la visée de connivence du DJS. Le journaliste introduit volontairement dans son discours les questions rhétoriques,

3 L'interrogation est fictive quand aucune réponse n'est attendue, ayant un effet semblable à celui d'une exclamation. Alors que « [1]'interrogation oratoire est une interrogation fictive qui donne à entendre qu'il faut admettre comme évidente la réponse contredisant la question » (Le Bon usage $2016: 387$ ). 
car le fait de poser une question, quoique l'action d'y répondre ne soit pas possible, représente déjà une forme d'adresse. Le lecteur se sent sollicité, même sans pouvoir ni donner ni souvent connaître la réponse.

\subsection{Niveau discursif}

$\mathrm{Au}$ niveau discursif, des marques conversationnelles sont introduites dans le DJS, relevant d'une logique de la conversation strictement parlée (évoquant, par exemple, des tours de parole) ainsi que de l'oralité.

Les expressions déictiques renvoient à la situation de communication immédiate : elles permettent d'identifier les participants d'un acte communicationnel donné, le moment et le lieu de l'énonciation. Différentes parties du discours fonctionnent en déictiques, à savoir les pronoms personnels et démonstratifs, les déterminants démonstratifs, les adverbes de lieu et de temps. Dans l'optique de la connivence journaliste-lecteur dans le DJS, il vaut nous attarder sur la classe des pronoms. Par leur rôle déictique, les pronoms personnels de la première (je, nous) et de la deuxième personnes (tu, vous), complétés ponctuellement par le pronom on lorsque celui-ci se réfère à nous, permettent d'identifier les (inter)locuteurs selon la situation de communication donnée.

Dans le discours journalistique, il est rare que l'auteur se déclare. Quand il le fait dans le DJS, ce n'est pas non plus par un je $e^{4}$ mais en s'intégrant à l'indéfini on $(12,13)$. Celui-ci reste d'ailleurs ambigu; il pourrait tout autant s'agir d'un pronom indéfini que d'un substitut de nous. Le DJS français semble en effet mettre à profit la double interprétation de on qui y garde un indice de pluralité.

(12) Si l'on dissèque la situation avec la froideur d'un bistouri, la possibilité d'une victoire à Paris s'est sans doute envolée, même si c'est un objectif que le Franc-Comtois n'a jamais avoué. (EQ 16.7.19 Cyclisme)

(13) «Vous savez, je me défends pas mal dans les chronos «, dit-il posément. Et on le croit. (EQ 19.5.19 Cyclisme)

Signaler la présence de l'allocutaire d'une manière directe est un fait occasionnel. Vous y apparaît, comme dans l'exemple (14) où l'on s'adresse directement au(x) lecteur(s). S'il est vrai que vous exclut la possibilité d'avoir un nous, un vous et moi, cela n'amoindrit pas son aspect interactionnel. Dans (14), il y a en même temps l'occasion d'observer la présence plus ou moins dévoilée des deux côtés de l'acte de communication : l'allocutaire

4 Le journaliste ne se dévoile en tant qu'énonciateur que dans des genres dits interprétatifs. Dans notre corpus, nous n'avons pu relever aucune occurrence d'un pronom personnel à la première personne qu'émettrait le journaliste et non pas un interviewé. 
(indéfini) admis par vous, et le locuteur attesté par un on qui y semble à cheval entre un emploi générique et un emploi de on se substituant à nous.

(14) Les Championnats du monde de natation viennent de prendre fin à Gwangju, en Corée du Sud. Si la superstar américaine Caeleb Dressel a encore plus assis sa domination sur la discipline, avec six titres, il y a eu d'autres coups d'éclat... et quelques éclaboussures. Si vous n'avez rien suivi, pas de panique, on vous fait un résumé. (M 2.8.19 Natation)

Dans l'extrait (15), on opte pour mettre en relief le lecteur, tandis que le journaliste lui-même se trouve dans une description animée du déroulement du Tour de France 2019. Par son style d'écriture, l'auteur incite le récepteur distancé physiquement à s'imaginer sur place. Moyennant un style inclusif, la réitération du pronom vous et des phrases interrogatives, il parvient à donner lieu à une ambiance de connivence, de participation. Celleci est finalement couronnée dans la dernière question où, pour conclure, on est convié au sentiment d'excitation partagé grâce à vous aussi.

(15) Vous le sentez, ce petit frisson, ce filet d'émotions qui vous met les poils au garde-à-vous, vous fait trembloter la carcasse et chauffer l'unité centrale ? Vous le voyez, Julian Alaphilippe, tenter d'aller cueillir le Maillot Jaune à Épernay ou Colmar, lundi ou mercredi prochain ? Vous l'imaginez, Warren Barguil, déchirer la foule dans les cols des Pyrénées avec son tout neuf paletot bleu-blancrouge ? Et Romain Bardet et Thibaut Pinot porter le combat jusqu'au bout de la troisième semaine, le maillot ouvert sur leur petit poitrail de moineau, bouche entrebâillée comme une carpe en recherche d'oxygène, toutes dents dehors ? C'est bon, vous aussi vous avez les connexions qui grésillent ? (EQ 6.7.19 Cyclisme)

Une conversation abonde en marqueurs discursifs qui, sans contribuer au contenu de l'énoncé, " possèdent un sens procédural, c'est-à-dire qu'ils donnent des informations à l'interlocuteur sur la façon de percevoir et d'interpréter un énoncé » (Grevisse et Da Silva-Genest $2018: 42$ ). Issus de diverses classes grammaticales, ils sont plurifonctionnels et donc à interpréter selon le contexte. Ils ont pour fonction d'orienter et d'ordonner le discours (tours de paroles, ouverture et clôture de l'énoncé), d'établir un rapport interactionnel et de maintenir l'attention de l'interlocuteur, de laisser transparaitre le rapport $\mathrm{du}$ locuteur envers le contenu propositionnel. Un tel effet est produit par le marqueur discursif bon en français où «bon portant à droite est fréquemment associé à un mouvement de concession » (Auchlin 1981 : 98). Dans (16), on peut observer qu'il est bel et bien dispensable vis-à-vis du contenu ; la deuxième phrase ne perdrait rien au niveau propositionnel sans bon. 
(16) « On a chanté vite fait 'on est en finale' dans le bus en rentrant, mais c'est tout «, lâche Alexandra Lacrabère, voix rocailleuse et petits yeux après s'être endormie à 3 heures du matin passées. Bon, les Françaises n'allaient pas non plus lancer une chenille samedi lors de leur visite de l'AccorHotels Arena vide, quand elles ont dû répondre aux médias une demi-heure durant. (EQ 16.12.18 Handball)

Dans la phrase figée C'est bon (17), l'adjectif est sans valeur propositionnelle mais conclut le discours d'une façon oralisée. Ce type d'insérée signale clairement un effet voulu autre que strictement informatif ; l'insertion d'un marqueur de discours à l'écrit établit surtout un parallèle à l'interaction verbale.

(17) C'est bon, vous aussi vous avez les connexions qui grésillent ? (EQ 6.7.19 Cyclisme)

Dans leur rôle de structurer le discours, les marqueurs discursifs servent « de ponctuation du discours oral » (Riegel et al. 2001 : 36). L'exemple (18) illustre l'emploi de voilà où il sert à clore le passage narratif. Un marqueur de discours polyvalent, voilà, $\mathrm{y}$ appuie l'imitation du commentaire en direct, créée moyennant des phrases courtes, découpées, et des formes verbales au présent.

(18) Il sourit, ferme les yeux, semble un brin circonspect. Soulagé, surtout. Le speaker s'égosille. Voilà, c'est fait. (EQ 22.6.19 Natation)

(19) Après s'être procuré deux balles de break, le protégé de Thomas Johansson s'emparait de l'engagement du Serbe, malmené, à 3-3. Seulement voilà, le $n^{\circ} 1$ mondial n'est pas là par hasard. (EQ 10.7.19 Tennis)

Mentionnons encore les marqueurs discursifs enfin (20) et alors (21) qui témoignent davantage d'une tendance à rendre le DJS plus conversationnel.

(20) Crystal Dunn, comme la Brésilienne Tamires, attaque beaucoup mieux qu'elle ne défend, ce qui peut donner des idées à Kadidiatou Diani ou à d'autres, dans l'espace entre la latérale et la charnière. Enfin, Kelley O'Hara, notamment, ne maîtrise pas toujours sa fougue. À la $72^{\mathrm{e}}$ minute, face à la Suède, elle est passée tout près de provoquer un penalty et d'écoper d'un deuxième carton jaune pour un coup de tête involontaire. (EQ 26.6.19 Football)

(21) Alors bien sûr, ce deuxième but en deux matches ne suffira pas pour racheter «Ney» aux yeux des plus fervents Ultras parisiens. (F 23.9.19 Football) 
Nous avons déjà observé comment le DJS prétend à l'interaction par la succession question-écho + réponse. La stratégie de ne laisser entendre qu'une part de la conversation consiste aussi à employer des expressions d'affirmation, créant ainsi un effet de conversation en cours. Dans (22), il semble même que le journaliste soit entré dans un triangle communicatif : il jongle entre une « interaction » avec son interviewé dont il rapporte les paroles à l'interlocuteur-lecteur, réagissant visiblement aux questions de ce dernier. L'accord mutuel dans (23) est renforcé par un oui suivi de la phrase c'est vrai, qui acquiesce aux propos de l'interlocuteur. Dans tous les exemples, on sent d'ailleurs s'annoncer une opposition plus ou moins prononcée par la suite.

(22) Oui, l'entraîneur allemand du PSG estime que le match laborieux de son équipe « peut donner confiance » pour la suite. Oui, le dénouement rappelle qu' « il est nécessaire de combattre, de toujours croire que c'est possible ». (M 27.11.19 Football)

(23) Oui c'est vrai, l'équipe de France a fait preuve d'une admirable continuité en gardant sa place sur la troisième marche du podium mondial pour sa première compétition sans un seul des membres de la fameuse génération « 82 » (Parker, Diaw, F. Pietrus), celle qui l'a habituée à fréquenter les plus hautes cimes. (EQ 16.9.19 Basketball)

\section{5}

CONCLUSION

Dans le présent article, nous avons étudié ces stratégies conversationnelles qui, dans le DJS français, contribuent par leur allusion à une interaction à la stratégie de connivence entre le journaliste sportif et ses lecteurs. Nourrie par des sentiments de cohésion qu'inspire le sujet sportif, cette connivence se traduit souvent dans le DJS par une quête d'informalité. Nous y avons ajouté l'analyse de l'aspect interactionnel afin de comprendre quelle approche, informelle ou interactionnelle, est plus utilisée et donc plus productive pour maintenir une impression de communauté dans le DJS.

L'analyse qualitative du DJS français aux niveaux lexical, syntaxique et discursif a montré qu'on essaie d'établir un lien de connivence avec les lecteurs, avant tout par l'emploi du lexique informel. Couplé au procédé familier d'apocope, le lexique familier semble y être intégré naturellement, cherchant ainsi un contact informel avec le lecteur. À la suite de la même logique, les marqueurs discursifs et les pronoms à valeur déictique interpellent le lecteur en tant qu'allocutaire et interlocuteur fictif. Caractéristiques de l'oral plutôt que de la langue écrite et puisqu'ils sont associés à un style informel, les marqueurs discursifs sont considérés comme des éléments à bannir de la norme. Parus dans le DJS, ils ne contribuent donc pas autant à sa structuration qu'ils servent à oraliser le discours. 
C'est au niveau syntaxique qu'on peut s'apercevoir d'une volonté interactionnelle, car l'aspect oralisant y est en arrière-plan ou pas inclus. Nous avons constaté l'emploi de la phrase interrogative à visée non-informative, notamment celui de la question-écho qui permet d'introduire dans le discours des traces d'un dialogue imaginaire. Le journaliste joue ainsi le rôle d'intervenant dans une conversation, exprimant son doute ou de l'incrédulité, voire réagissant lui-même à la « question » par une réponse. Une dynamique dialogique s'installe donc dans le DJS. D'autres interrogatives partent d'une vraie lacune d'informations. Il semble que dans le cadre d' « un dialogue fictif », effectivement, ces formes d'interrogation partielle incitent le lecteur, sinon à prendre la parole réellement, du moins à y réagir par la pensée.

Dans la perspective d'établir un lien entre les journalistes et les lecteurs dans le DJS, la stratégie principale qui consiste à imiter la conversation semble reposer sur l'introduction d'éléments relevant d'un style oralisé, et non de l'échange. Lorsqu'elle est appliquée au DJS, la notion de conversationnalisation s'appuie notamment sur les stratégies générant l'ambiance détendue d'une interaction verbale informelle. De ce fait, elle ne relève pas autant de l'imitation de la conversation en tant qu'interaction, mais surtout des circonstances de la communication verbale informelle. C'est-à-dire que la connivence dans le DJS est étroitement liée aux indices d'une ambiance conviviale et détendue, entre les connaisseurs sportifs non hiérarchisés. Il importe de faire référence à un sentiment de partage et de communauté, ce qui est réalisé par différents moyens linguistiques évoqués au cours de l'analyse. Grâce aux tentatives de contact, le lien journaliste-lecteur est établi, tandis que les traits de l'informalité mettent en place une impression de communauté égalitaire.

La présente analyse suggère des pistes à creuser dans l'étude des stratégies conversationnelles dans le DJS. Couplée à une analyse qualitative et quantitative sur un corpus plus large, elle permettrait de mieux comprendre les procédés linguistiques que l'on entreprend pour construire le sentiment de partage et de connivence dans le DJS.

\section{BIBLIOGRAPHIE}

ANTOINE, Fabrice (2000) Dictionnaire français-anglais des mots tronqués. Louvainla-Neuve : Peeters.

AUCHLIN, Antoine (1981) Réflexions sur les marqueurs de structuration de la conversation. Études de Linguistique Appliquée 88, 88-115.

BANGE, Pierre (1983) Points de vue sur l'analyse conversationnelle. DRLAV. Documentation et Recherche en Linguistique Allemande Vincennes 29, 1-28.

BENTOUNSI, Ikram A. (2017) La question rhétorique et l'exclamation dans le discours journalistique de la presse écrite francophone algérienne. Langues \& Usages 1, 90-101. 
BOYER, Henri, Médias, Culture, Société (Cours EAD). Montpellier : Université de Montpellier 3.

CHOVANEC, Jan (2008a) Enacting in Imaginary Community: Infotainment in on-line minute-by-minute sports commentaries. In : LAVRIC Eva/Gerhard PISEK/Andrew SKINNER/Wolfgang STADLER (éds.), The Linguistics of Football. Tübingen : Gunter Narr Verlag Tübingen, 255-268.

CHOVANEC, Jan (2008b) Focus on form: foregrounding devices in football reporting. Discourse \& Communication 2/3, 219-242.

CHOVANEC, Jan (2009) Simulation of Spoken Interaction in Written Online Media Texts. Brno Studies in English 35/2, 109-127.

DELANEY, Tim (2015) The Functionalist Perspective on Sport. In : R. Giulianotti (éd.), Routledge Handbook of Sociology of Sport. London : Routledge, 18-28. Le 2 mai 2020. https://www.routledgehandbooks.com/doi/10.4324/9780203404065.ch3.

DEREZE, Gérard (2009) Le Récit sportif hautement médiatisé : quelques réflexions. Les Cahiers du journalisme 19, 90-99.

FAIRCLOUGH, Norman (1992) Discursive Practices of Consumerism. Le 2 mars 2021. Fairclough, Discursive Practices of Consumerism.pdf.

FOWLER, Roger (1991) Language in the News: Discourse and Ideology in the Press. London: Routledge.

GREVISSE, Maurice/Christine DA SILVA-GENEST (2018) Le Grevisse de l'orthophoniste. Louvain-la-Neuve : De Boeck Supérieur.

HERMAN, Thierry (2008) La connivence entre le journaliste et son lecteur. Un lieu d'échange entre sciences du langage et de la communication. Marcel Burger (éd.), L'analyse linguistique des discours des médias : apports, limites et enjeux. Québec : Nota Bene, 183-206.

JACQUES, Francis (1981) L'interrogation, force illocutoire et interaction verbale. Langue française 52, 70-79.

LEWANDOWSKI, Marcin (2012) The language of online sports commentary in a comparative perspective. Lingua posnaniensis LIV/1, 65-76.

PREOTEASA, Gigel (2017) Approches discursives du commentaire footballistique en français et en roumain entre presse papier et presse numérique. Thèse de doctorat. Université Bourgogne Franche-Comté.

RIEGEL, Martin/Jean-Cristophe PELLAT/René RIOUL (2001) Grammaire méthodique du français. Paris : Presses Universitaires de France.

STEVENSON, Cristopher L./John E. NIXON (1972) A Conceptual Scheme of Social Functions of Sport. Sportwissenschaft 2, 119-132.

TRAVERSO, Véronique (2003) Les genres de l'oral : le cas de la conversation. Les genres de l'oral. Lyon. Le 3 juin 2020. https://halshs.archives-ouvertes.fr/halshs-01001725/ file/Trav_GenreOral_Conversation.pdf. 
VANOUDHEUSDEN, Romain (2010) Stéréotypes et variation sémantique dans un corpus de presse sportive en anglais et en français (thèse de doctorat). Université de Poitiers.

\section{ANNEXE : LISTE DES SOURCES D'EXEMPLES}

L'Équipe (consultable sur : https://www.lequipe.fr/)

1. EQ 16.12.18 Handball : «Le rêve bleu de l'équipe de France, en finale de son Euro contre la Russie », consulté le 16 décembre 2018.

2. EQ 31.12.18 Rugby : « Bordeaux-Bègles finit l'année en trombe contre le Racing 92 », consulté le 31 décembre 2018.

3. EQ 19.5.19 Cyclisme : " Giro : les prétendants vont devoir se découvrir sur la $9^{\mathrm{e}}$ étape », consulté le 19 mai 2019.

4. EQ 22.6.19 Natation : " Meeting des Sept Collines : Florent Manaudou, comme un ouragan », consulté le 22 juin 2019.

5. EQ 26.6.19 Football : « Coupe du monde 2019 : comment jouent les États-Unis, adversaires des Bleues en quarts », consulté le 26 juin 2019.

6. EQ 28.6.19 MotoGP : « MotoGP : requiem pour Valentino Rossi », consulté le 28 juin 2019.

7. EQ 30.6.19 Basketball : « Championnat d'Europe : les Bleues ne doivent pas se rater contre la Suède », consulté le 30 juin 2019.

8. EQ 6.7.19 Cyclisme : « Comme un frisson au moment du départ du $106^{\mathrm{e}}$ Tour de France », consulté le 6 juillet 2019.

9. EQ 10.7.19 Tennis : «Wimbledon : Novak Djokovic écrase David Goffin et rejoint les demi-finales », consulté le 10 juillet 2019.

10. EQ 16.7.19 Cyclisme : «Tour de France : un coup de bordure ruine le rêve de Thibaut Pinot lors de la $10^{\mathrm{e}}$ étape », consulté le 16 juillet 2019.

11. EQ 16.9.19 Basketball : « Deuxième titre mondial pour l'Espagne, victorieuse de l'Argentine », consulté le 16 septembre 2019.

12. EQ 23.9.19 Volleyball : «Euro : mardi, c'est la fête des voisins entre l'Italie et la France », consulté le 23 septembre 2019.

13. EQ 20.10.19 Rugby : «Bleus : toujours sur courant alternatif », consulté le 20 octobre 2019.

14. EQ 1.11.19 Tennis : «Rolex Paris Masters : Novak Djokovic rejoint Grigor Dimitrov en demi-finales », consulté le $1^{\text {er }}$ novembre 2019.

15. EQ 14.11.19 Basketball : «Eurocoupe : le réveil fracassant de Monaco », consulté le 14 novembre 2019. 
Le Figaro (consultable sur https://sport24.lefigaro.fr/)

16. F 4.1.19 Handball : « Les Experts débutent leur préparation à Créteil », consulté le 4 janvier 2019.

17. F 6.7.19 Football : « CAN 2019 : le Sénégal tient son rang face à l'Ouganda et retrouvera le Bénin », consulté le 6 juillet 2019.

18. F 23.9.19 Football : «Impitoyable, Neymar Jr refait le coup contre Lyon », consulté le 23 septembre 2019.

19. F 8.10.19 Rugby : «XV de France : pas d'impasse face à l'Angleterre », consulté le 8 octobre 2019.

20. F 20.11.19 Tennis : « Dans la douleur, la doublette Mahut-Herbert offre la victoire aux Bleus », consulté le 20 novembre 2019.

Le Monde (consultable sur https://www.lemonde.fr/sport/)

21. M 5.6.19 Tennis : «Roland-Garros : dans le tableau féminin, l'hécatombe des têtes de séries se poursuit », consulté le 5 juin 2019.

22. M 2.8.19 Football : « CAN 2019 : l'Algérie remporte le deuxième titre de son histoire », consulté le 2 août 2019.

23. M 2.8.19 Natation : "Ce qu'il faut retenir des Mondiaux de natation : des records et des remous », consulté le 2 août 2019.

24. M 4.11.19 Football : "Ligue 1 : Lyon s'impose in extremis, Angers deuxième du classement », consulté le 4 novembre 2019.

25. M 27.11.19 Football : «Le PSG, un point d'honneur à ne pas perdre », consulté le 27 novembre 2019.

POVZETEK

\section{KONVERZACIONALIZACIJA KOT DISKURZIVNA STRATEGIJA V FRANCOSKEM NOVINARSKEM DISKURZU O ŠPORTU}

Ustvarjanje in ohranjanje navidezne vezi med novinarjem in bralci je ena od komunikacijskih strategij novinarskega diskurza o športu, kakršen se pojavlja v tiskanih medijih. Novinarski diskurz je namreč monologalen in ima kot tak določene komunikacijske omejitve. Eno od ključnih predstavljata pomanjkanje oziroma nezmožnost neposrednega stika med piscem in njegovimi naslovniki. Po drugi strani ima šport znatno kohezivno funkcijo, kot tak pa krepi občutek pripadnosti navidezni (športni) skupnosti tudi preko novinarskega diskurza. Navidezna vez je torej strategija novinarskega diskurza o športu, kako premostiti diskurzivno vrzel med novinarji in bralci, povrhu 
pa okrepiti vtis povezanosti, ki jo navdihuje športna tematika. V novinarskem diskurzu o športu se pojavlja v obliki različnih jezikovnih sredstev, med drugim konverzacijskih elementov. S pomočjo kvalitativne analize izbranih člankov francoskega športnega tiska smo preverili, preko katerih jezikovnih sredstev poteka oponašanje pogovornih elementov in kako slednje prispeva k uresničevanju strategije navidezne vezi. Pod drobnogled smo vzeli neformalne in interaktivne konverzacijske značilnosti. Analiza je pokazala, da se konverzacionalizacija izraža predvsem v neformalnem značaju diskurza, in sicer na besedni in diskurzivni ravni. Pogovorno besedišče in apokopa ter pojavljanje diskurzivnih označevalcev namigujejo na neuradno komunikacijsko situacijo, ta pa vodi k občutku navidezne športne skupnosti. Ta vtis je na skladenjski ravni podkrepljen z retoričnimi vprašanji in drugimi različicami vprašalne povedi, ki naslavljajo bralca in v novinarski diskurz o športu vnašajo interaktivno dinamiko dialoga med navideznimi sogovorci. V francoskem športnem tisku sta torej prisotna tako neformalni kot interaktivni pristop v oponašanju konverzacije. Oba $\mathrm{v}$ diskurzu prispevata $\mathrm{k}$ vtisu neformalnega vzdušja med novinarjem in naslovniki ter $\mathrm{k}$ občutku povezanosti med športnimi navdušenci.

Ključne besede: šport, diskurz, strategije, navidezna vez, konverzacionalizacija

\section{ABSTRACT}

\section{IMITATION OF CONVERSATION AS A DISCURSIVE STRATEGY IN THE FRENCH SPORTS NEWS DISCOURSE}

Creating and maintaining an imaginary bond between the journalist and readers is a communicational strategy of the written sports news discourse. Since news discourse is monologal, it has specific communicational constraints that it seeks to overcome, and the lack of direct contact between the authors and their readers is a key hurdle to cross. Additionally, sport has a notable cohesive function, and as such produces a sense of a sports community even when taken as a news subject. The imaginary bond is therefore a strategy for overcoming the discursive gap between the journalist and their readers, while at the same time reinforcing the impression of community that the subject of sports draws on. In the sports news discourse this strategy is translated through different linguistic means, such as conversational elements. Based on selected articles from the French written sports news, a qualitative analysis of texts enabled us to observe the linguistic means through which the imitation of conversation contributes to the discursive strategy of bonding. The analysis is focused on informal and interactive conversational features. It shows that such conversationalization is particularly apparent in the informal character of the discourse on the lexical and discursive levels. This adds to the seeming informality of the communicational situation, and therefore to the readers' sense of an imaginary sports community. On the syntactic level this is reinforced by interactional elements such as rhetorical questions and other interrogatives that address the reader as an interlocutor and echo the dynamics of a dialogue. Both conversational aspects, as used in the 
French sports news discourse, contribute to a sense of informal atmosphere and community bond among sports enthusiasts.

Keywords: sport, discourse, strategies, imaginary bond, conversationalization 